

\title{
Wissenstransfer in Kooperationen zwischen Universitäten und Museen
}

Kathrin Anzinger

Museen sind wie Universitäten sowohl Orte der Wissensproduktion als auch der Wissensvermittlung. Beide Institutionen haben einen Forschungs- und Vermittlungsauftrag. Darüber hinaus zählen das Sammeln und Bewahren sowie das Ausstellen zu den Aufgaben der Museen. Diese Aufgabenfelder bieten ein vielfältiges Potential für Wissenstransfer, woraus sich Gründe ableiten lassen, die für Kooperationen zwischen Universitäten und Museen sprechen:

\section{Universitäre Forschung in der Öffentlichkeit sichtbar machen}

Museen bieten ein geeignetes Umfeld, um der Bevölkerung Einblicke in die universitäre Forschung zu geben und universitäres Wissen nachvollziehbar zu vermitteln.

Dies zum einen deshalb, weil Museen sich an eine breite Öffentlichkeit richten und sie als öffentlich zugängliche Orte wahrgenommen werden. Ihr Anliegen ist, durch Ausstellungen Besucher*innen jeden 
Alters, jeder Herkunft, mit unterschiedlichem Bildungshintergrund oder beruflicher Tätigkeit zu erreichen und ihnen einen angenehmen Museumsbesuch zu ermöglichen.

Zudem fordert die Aufgabe, Forschung einem breiten Publikum näherzubringen, viel Zeit, Können und Praxiserfahrung, da komplexe wissenschaftliche Inhalte in eine alltägliche Sprache übersetzt werden müssen, ohne dabei auf ihre korrekte Darstellung zu verzichten. Viele Museen verfügen über Vermittlungspersonal, das für diese Aufgabe geschult ist. Museumspädagog*innen zeichnen sich durch zielgruppenspezifische, didaktische Fertigkeiten und spezifisches Fachwissen aus. Ihre Vermittlungsarbeit baut auf den in der Ausstellung gezeigten Objekten auf, welche betrachtet, berührt oder ausprobiert werden können. Durch objektbezogenes und experimentelles Lernen wird Wissen für die Besucher*innen erfahrbar.

\section{Zugang zu aktuellen}

\section{Forschungsergebnissen von Universitäten schaffen}

Das Ausstellen und Vermitteln von universitärer Forschung in Museen dient nicht ausschließlich dem Nutzen der Universitäten. Für die Museen bedeutet es - im besten Fall kontinuierlich - Zugang zu aktuellen Forschungsergebnissen zu erhalten, die in Ausstellungen einfließen, neue Ausstellungskonzepte begründen oder die eigene Forschungsarbeit ergänzen.

\section{Universitäre Lehre um objektbezogenes Lernen bereichern}

Objektbezogenes und experimentelles Lernen, wofür Museen durch den Sammlungsbestand und seine Museumsgüter die idealen Voraus- 
setzungen bieten, ist nicht nur für die zielgruppenspezifische Vermittlungsarbeit von Vorteil, sondern stellt auch für die universitäre Lehre eine Bereicherung dar. Selbst wenn Objekte im Sinne des Bewahrens nicht berührt werden dürfen, so ist das Betrachten eines Originals immer aufschlussreicher als das Betrachten eines Abbildes. Das Objekt kann aus unterschiedlichen Blickwinkeln wahrgenommen werden, Details können ,unter die Lupe' genommen werden, Materialbeschaffenheit und Techniken werden ersichtlich und das Auge wird geschult.

Abgesehen davon verfügen Museen oftmals über alte, seltene oder einmalige Maschinen und Instrumente, die von Studierenden ausprobiert werden können, wodurch ihnen Funktionalität, Techniken und fachspezifische Fertigkeiten vermittelt werden. Zugleich erfahren die Studierenden über deren Geschichte und Entwicklung bis hin zur Moderne. Archive, in denen alte Muster, Modelle oder Skizzen aufbewahrt werden, geben zusätzliche Impulse.

Generell bieten Museen die Gelegenheit, die Hörsäle und Seminarräume der Universitäten zu verlassen und einen Teil der Lehre auszulagern.

\section{Fachspezifisches Wissen von Universitäten für Museen anbieten}

Universitäten und ihre unterschiedlichen Fachgebiete bieten Museen einen Fundus an fachspezifischen Kompetenzen, auf die insbesondere dann zurückgegriffen werden kann, wenn personelle Ressourcen fehlen oder das notwendige Fachwissen nicht vorhanden ist. Die Band- 
breite an Möglichkeiten ist groß und bezieht sich auf alle musealen Aufgabenfelder: So unterstützen Universitäten bei der...

... Aufarbeitung und Inventarisierung des Sammlungsbestandes.

... Konservierung und Restaurierung von Museumsgütern.

... inhaltlichen Aufarbeitung von Ausstellungen.

... räumlichen und medialen Ausstellungsgestaltung.

... Erstellung von Ausstellungspublikationen.

... Entwicklung von zielgruppenspezifischen Vermittlungsprogrammen.

Universitäten haben dadurch die Möglichkeit, neue Erkenntnisse, Verfahren und Techniken in der Praxis zu erproben und Wissen über aktuelle Bedürfnisse und Anforderungen im Praxisfeld zu generieren. Die Einbindung von Studierenden erlaubt darüber hinaus einen hohen Praxisbezug in der universitären Lehre.

\section{Fachspezifisches Wissen von Museen für Universitäten anbieten}

Umgekehrt verfügen Museen über spezifisches Wissen und Fachkompetenzen, die für Studierende bestimmter Studienrichtungen von Relevanz sind, da das Museum für sie nach Abschluss des Studiums ein potenzielles Betätigungsfeld darstellt. Indem z.B. kaufmännische und künstlerische Museumsleiter*innen, -pädagog*innen oder -kurator*innen in die universitäre Lehre einbezogen werden, lernen die Studierenden über das Betriebssystem ,Museum', die künstlerische und kuratorische Praxis in Museen oder über museumsspezifische Vermittlungsmethoden, die auch für andere berufliche Tätigkeiten im Bereich der Pädagogik relevant sein können. Praktikumsplätze, die den Studierenden von Museen angeboten werden, unterstützen dies darüber hinaus. 


\section{Forschung durch Studierende ermöglichen}

Die Sammlungsbestände von Museen sind außerdem für Abschlussarbeiten und Dissertationen von Studierenden interessant. Voraussetzung ist, dass den Studierenden der Zugang zu den Depots gewährt wird und sie dort regelmäßig arbeiten können. Im Gegenzug können die Museen auf die im Zuge der Arbeiten gewonnen Erkenntnisse zurückgreifen und für ihre Zwecke nutzen. Dies ist vor allem dann relevant, wenn die Museen bereits bei der Themenfindung einbezogen werden.

\section{Forschung gemeinsam betreiben}

Forschungsaktivitäten, die von Universitäten und Museen gemeinsam durchgeführt werden, können über den Zweck der Ausstellungskonzeption hinausgehen und als mehrjährige Forschungsprojekte mit interdisziplinärem Charakter angelegt sein. Interdisziplinäre Forschung ist zwar eine herausfordernde Aufgabe, bietet aber stets das Potenzial für innovative Erkenntnisse, da der Austausch und die Integration von Wissen unterschiedlicher Fachbereiche neue Sichtweisen und $\mathrm{Zu}$ gänge eröffnet.

Diese und andere Gründe, die für die Zusammenarbeit von Universitäten und Museen sprechen, sind Teil eines Manuals, das bei der Anbahnung und Durchführung von Kooperationen zwischen Universitäten und Museen Hilfestellung bieten soll.

Das Manual gibt zunächst einen Überblick über die Aufgabenfelder von Universitäten sowie Museen und legt Gründe, die für aber auch gegen Kooperationen zwischen Universitäten und Museen sprechen, dar. In weiterer Folge werden die Phasen einer Kooperation - von der Vorbereitung, über die Planung, bis zur Durchführung - und die den 
Phasen zugeordneten Arbeitsschritte dargestellt. Ergänzend finden sich im Manual Zitate von Museumsvertreter*innen und Universitätsmitarbeiter*innen, nationale und internationale Beispiele für Kooperationen zwischen Universitäten und Museen sowie eine Checkliste, die wichtige Fragen und To Do’s zusammenfasst.

Erstellt wurde das Manual auf Grundlage recherchierter Literatur über Kooperationsmanagement sowie Leitfäden und Guides mit Fokus auf Kooperationen von Museen mit Einrichtungen im Bereich der höheren Bildung und des Gemeinwesens, die es zum damaligen Zeitpunkt so nur im englischsprachigen Raum gab. Zudem fanden im Juni 2016 drei Gruppendiskussionen statt, zu denen Vertreter*innen regionaler Museen in Oberösterreich und Mitarbeiter*innen der Kunstuniversität Linz eingeladen wurden. Gemeinsam wurden Gründe für und gegen Kooperationen zwischen Universitäten und Museen gesammelt sowie die notwendigen Arbeitsschritte in den jeweiligen Phasen einer Kooperation diskutiert.

Anstoß für das Manual gaben die Ergebnisse eines Forschungsprojektes über Kooperationsaktivitäten der Kunstuniversität Linz mit Museen in Oberösterreich, veranlasst durch den Umstand, dass das Potenzial möglicher Anknüpfungspunkte zwischen den Instituten und Abteilungen der Kunstuniversität Linz und den oberösterreichischen Museen im Großraum Linz zwar nicht brach lag, aber, vor allem in Bezug auf regionale Museen, noch stärker genutzt werden hätte können. Demnach zielte die Forschungsarbeit darauf ab,

.. e einen Überblick über die Museumslandschaft in Oberösterreich zu geben.

... die Aktivitäten der oberösterreichischen Museen in Hinblick auf die vier musealen Aufgabenfelder zu ergründen.

... vergangene und bestehende Kooperationsprojekte der Kunstuniversität Linz mit oberösterreichischen Museen darzustellen. 
... Voraussetzungen und Herausforderungen im Zusammenhang mit Kooperationen und deren Anforderungen zu identifizieren.

.. das bestehende Potenzial für zukünftige Kooperationen zu ergründen.

.. mögliche Schritte in Richtung einer Intensivierung der Kooperationsaktivitäten abzuleiten.

.. $\quad$ einen Blick auf Kooperationstätigkeiten anderer (Kunst-)Universitäten über die Ländergrenzen hinweg zu werfen.

Die Auswertung von insgesamt 21 qualitativen, leitfadengesteuerten Interviews mit Leiter*innen und Mitarbeiter*innen von Museen in Oberösterreich, leitenden Mitarbeiter*innen der Kunstuniversität Linz sowie weiteren Vertreter*innen der oberösterreichischen Museumslandschaft ergab als einen möglichen Schritt zur Intensivierung der Kooperationsaktivitäten, die Erstellung eines Leitfadens. Dieser sollte präzisieren, wie das Vorgehen im Hinblick auf die Anbahnung und Durchführung von Kooperationen mit der Kunstuniversität Linz erfolgen soll. Das Manual wurde mit Bezug zu dieser Idee konzipiert, jedoch nicht spezifisch für die Kunstuniversität Linz, sondern allgemein für Universitäten und Museen, um einen breiteren Nutzen zu generieren.

Sowohl die Forschungsarbeit als auch das Manual wurden im Rahmen des Wissenstransferzentrums West erstellt und sind dort unter http://wtz-west.at/kooperationen universitaeten museen/ abrufbar. 